Alae ad celeriorem fugam thoraci affixae funt, aut cruftaceae, vaginantes, Elytra: aut molles, tenues, Alae: Alas ad clafirum determinationem introduxit Linné;

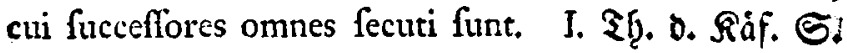
93. Degeer IV. Zaf. 2. Fig. 26.) Wemn Der Ftŭ̉get unter ber flügeldecfe liegt, ift er jebergeit boppelt jujam. men gefaltet, námlid, die Gpif̧e Deffelben a. b. c. iff jeberzeit umgeihhlagen, ju weldzem (Enbe fich in bem Saauptnerben bey a. ein gleidzfain po zu nennendes Cf̧ar: nier befindet, weldzes berweglid, ift. Ferner liegt auch Der 3 geeil bon d. bis e, ober Der feinete untere Fano bes Flügés, jederzeit zurủf gefd̨lagen. Degeer ḩat ben Jlügel oḩngefäţe fo borgeftellt.

\title{
Ert $\mathfrak{a} \mathfrak{i} \mathfrak{a n d}$

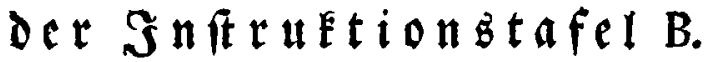

II. Die Jerglieserung cines Ttâfers aus ber Gattung Scarabaeus Linn. Mclolontha Fabr. *) Gen. Inf. p. 7 . Gen. 4. Melolontba. Os maxillis palpis. que quatuor.

Sigur I. ber Fopf von Dem befaniten Scarabaeus Fullo Linn. (Melolontha Fullo Fabr.) in naturtither Grópe ;

*) Şiet follte, ber Dronung beş fábricins nadh, etft bie

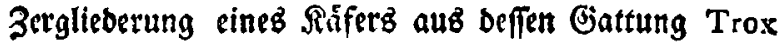
folgen, bie ifm junådift Scarabaeus ffeft; allein id Gabe weiter oben Eeite xiI. bereits ben Girund angeges ben, warum th fold)e vor biesmal nod nidht liefern tann. Bwar bin id babey, sinige biefer Siffer aufju= spelchent. 
Grdßke; a. a. Der Ropf, b. ber vordere faft bey allen $\mathfrak{A} r=$ tent biefer Battung Des Sabricius ausgefçweifte Rand, c. c. Die Augen, die ben Den mehreften $\mathfrak{A}$ rten bunt ges

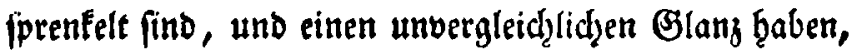

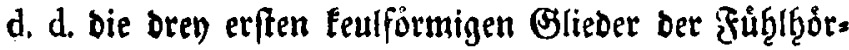
ner, e. e. bie fieben groß̧en Samellen, worin bie fühthl= Gơrner endigen. Fabr. Gen. Inf. p. 7. Gen. 4. An. tennae breues clauato lamellatae: articulo primo glo. bofo, craffiori. et Obf. 2. Antennarum claua in quibusdam lamellas feptem gerit. (Degeev IV. Kaf. IC. fig. 13.) *).

Sigur 2. ein wciblidges Sillylhorn yon Melolontha villofa Fabr. in anfef̧ulicher $\mathfrak{B e r g r o ̊ g e r u n g ; ~ a . ~ b a s ~ e r f e ~}$ långfte uno zugleid, ftálffite Ģlied, b. eill Darauf folgen, Des furjes runbes, c. eit abermaḩls langes aber fdzwa. cheres Stiteb als bas erfte, d. d. jween gleich großje etwas zulammen gedrudfe runde Blieder, c. Das aus fünf ver. fhiedentlich langen Eamellen beftegende Ende. Sàmmes liḑ̧e

weidhen, unb gu serfucten, ob id nod etliche innere Sheile beż Mrunbes unbeihäbigt herauż bringen wers be, allein id) stucifle faft baran, well ble Råfer felbfe alle febre flein, und bajjer (d)wer ju befandeln fino. Sringe id noch etwas berauz, fo will ich es noch aut Enbe auf biefer Tafel nadbolen.

7) Degeer bat von Melolontha Fabr. nidfts wetter vor"

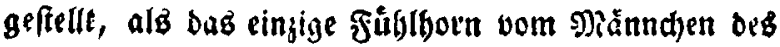
Melol. Follo, in natíllid)er Birofe, aUer feğ: undeutlid. 


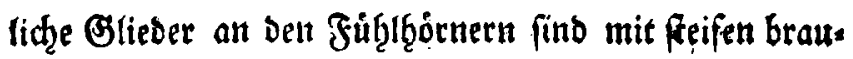

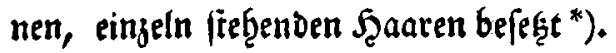

Sigur 3. Sie felhr vergróßerte Lippe von ber Melo. lontha folftitialis. Fabr. Gen. Inf. p. 7. Gen. 4. Labium porrectum, corneum, cordatum, apice fubemar ginatum. a. a. Der Brunotf̧eil berfelben, mit den b. b. Daran b̨ängenden Muskeln, c. c. Der Borderrand Derfel. ben,

*) Die Eamellen an ben Fäflgdruern wedfeln in Anfes Gung ibrer Alnzabl ungemein ab, fino aud ber Gird́be nadh fegr verfajieden, und meiftentheils bey ben siăs fern mănnliden BSefd)lecfits am lăngften. It) babe mir bafer jeţt nod (bei) fefre búfteren wsintertagen)

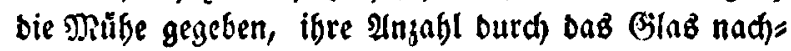
jujăblen, und bier ift bas, was id) geieben babe. Melolontha Fullo. mas. 7.lam. foem. 5. Mel. vulgaris mas. 7. lam. foem. 6. Mel. Solftitialis 3. lam. Mel. brunnea 3. I. Mel. argentea. 3. I. Mel. vitis 5. lam. Mel. Frifchii. 3. I. Mel, Spinipes. 3. I. Mel, berolinenfis. 3. Mel. puluerulenta. 3. Mel. Philantus. 3. Mel, horticola. 3: Mel. agricola. 3. Mel, farinofa. 3. Sd) bin aber nod nidht gewiß, ob id won ben mebreften Ilten bas mánnlidbe Ge(d)ledt bel) ber f̧and babe; fo viel

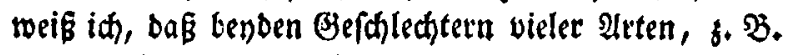
ber Mel. horticola, Frifchii u. a. m., bie id oft in ber Begattung gefangen habe, gleich grope uno gleid blele Blátter an den Fỉbl(hơrnern eigen find, uno id) bebals te mir vor, blervon bev Befdreibung ber einjelnen $A_{1}=$ ten btefer Gattung meine finftige Bemerfungen mits untgeilen. 
ben, weldhet fehte ausgebogen ift *), d. d. Die beubent binterften Frefspif̧en, wovon, weil fie etwas tief b̧inter Der Qippe cingelenft find, nut zwen) BJlieber fidztbar blei= ben. Sie ift übrigens, wie Sabricius fie befdereibt, Gerborrageno, Gornartig, faft Gerjförmig, über. baupt genommen, ein wenig ausyerandet, uno auf ben

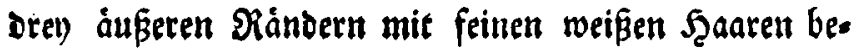
wadefert.

Sigur 4. Die vergrógerte \&uftere tintulabe. (Man. dibula Fabr. Gen. Inf. p. 7. Gen. 4. Mandibula bre. nìs, cornea, arcuata apice compreffa, acutiufcula, vis: dentata. a. a. Der lănglicht runbe Erundotheil, mit brenen baran ந̧ängenben wurgelformigen Musteln,

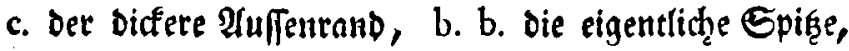
weldze burch einen tiefen Rerben gleid̨fam getheilt iff, und vier fleine వై úbrigen Drev aber ftumpf fino. Der obere Ţ̧eil ift fef̧r

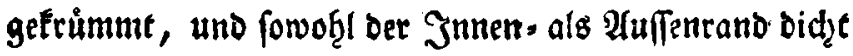
mit feinen Şaaren bewadyfen. Sabricius $\mathfrak{B e f d r e i b u n g}$

trifft

*) Sabricius Gen. Inf.p. 7. Gen. 4. ObC. I. fagt: Die Eippe ber Mel. folftitialis (ê) meler auzgerandet als die ber tubrigen Mdfer biefer Gattung. (Melolonshae folftitialis labium magis emarginatum quam in reliquis,) Sh) fann bier ntidfts beftátigen ober verneinen, weil id) nidit inebrere Irten unterfudjt babe, fo viel aber

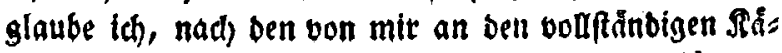
fern angeftellten thnterfuchungen, bef̧aupten fu fơnnen,

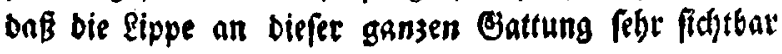
Ruछ̋geranbet iff. 


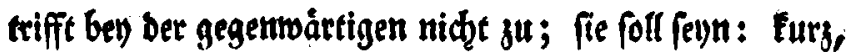
Ģornartig, gefrummt, an Der Spig̨e zufammen gedrůdft, jugefpişt, uno faum gejåḑnt. Die gegenoàrtige ḩat Deun entgegen aber vier beutlidz̨e כåănne.

Sigur 5. Die fef̧e vergrỏ Berte innere Zínnlabe (Maxilla Fabr. Gen. Inf. p. 7. Gen. 4. Maxilla cornea, breuis, rigida, fubarcuata, apice obtufa, multidentata:

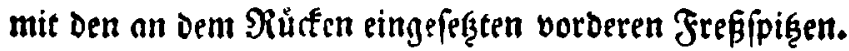
Diefe ift in ber Shat von fonderbarer Beftalt, uno fo viel ich an allen Kafern biefer Battung von autfentzee

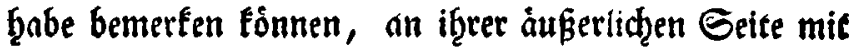
einer brebecfiditen $\mathfrak{P l a t t e}$ a. b. c. belegt, bie dicht mit fei-

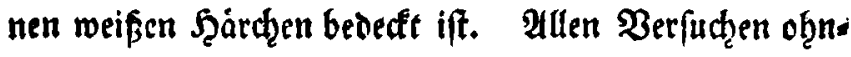
geaḑet, bat es mir nidzt gefingen wollen, diefe Platte fo fein abjulden, oa sie Rinnlabe vollfommen gang, uno unberfegrt geblieben wáre, ob/dyon idh oeutlid bee.

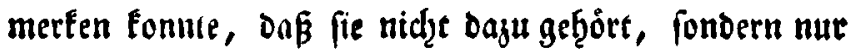

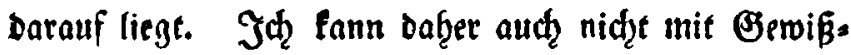
Geit beftimmen, ob bie Zapfen c. d. jur Rinnlade felbft, Diber nur zu der Darauf liegenden Platte, weldze vont

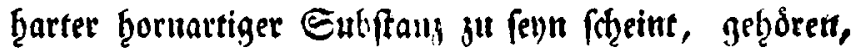

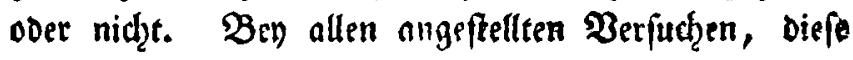

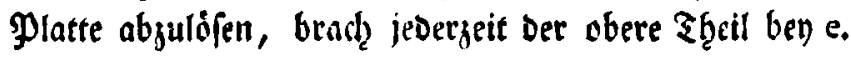
gerade Durd $a b$, fo dak cin fleines Stuff der Rinnlade bon biefer Platte entblöß̨t wurbe, wornus eb fich meinem Erachten nach ju ergeben fldzeint, Daß̧ Diefe Platte ein befondacer, aflenfalls abjulofender క̧̧eil fenn mulfe. f. f. Sft der Doppeite obere Şgeil der . Finulade, Der mir thidht fo ḩornartig zu fenn fągint, als das uibrige, unb

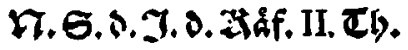


aus jween neben einander ftebenden lobis, einem grofise ren, vorn fugefpirzęen, und einem fleineten runden, befte: bet, bie gleidhfam in einer f̧ornartigen, glänzenden breits gebrúdten Sdzeibe fteffen. g. Die eine vordere Jreß̧. fpiz̨e. Sabricius fagt: Die innere Rimulade fey an ber

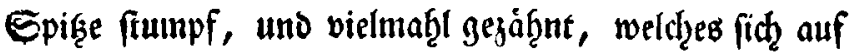
Die gegenwártige, wie bie abgebilbete Figur zeigt, eben nicht anwenden lápt.

Sigur 6. eine vergro̊ßzerte, atu vier ungleidyen Glies bern beftebende vordere Srefipitse. Fabr. Gen. Inf. p. 7. Gen. 4. Palpi inaequales filiformes; anteriores longiores, quadriarticulati: articulo primo tertioque breuiffimis, quarto longiori oblongo adhaerentes maxillae dorfo. a. c. Das erfte und dritte S̈lied, wels the faft gleidh grofi find, fo wie b. und d. Das zwente unb vierte.

Sigur 7. eine vergröperte bintere freffipitse, wor: an bas britte SGlieb a. ein wenig länger ift als bie benden erfteren. Fabr. Gen. Inf. p. 7. Gen. 4. pofteriores (palpi) breues, triarticulati: articulis fnbaequalibus labii parieti interiori inferti.

Sigur 8. ein vergro̊perter galljer Doroerfuf von Dem månnliden Melolontha Fullo. a. Die Şưfte ober

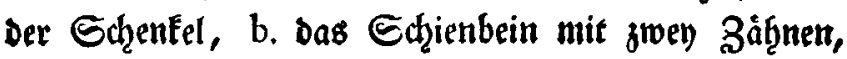
c. eine berweglidze, an glatten $\Re a n b e$ eingefegrte felre fteife দ̧ornartige Epiąe, d. Der eigentliche Fuß wöḩnlid), aus vier $\mathfrak{F u b b l a ̊ t t e r n ~ u n d ~ D e m ~ l e g ̧ t e n ~ l a n g e n ~}$ 


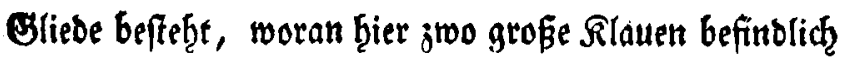
fino, bie unter fid nod eine feb̨r Eleine ḩaben. În

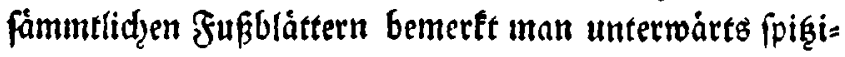

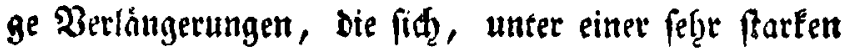

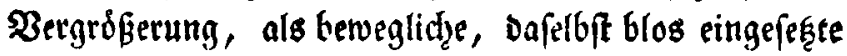
freife Spişen zeigett, of̧ngefefgr wie c. am Sḑienbein.

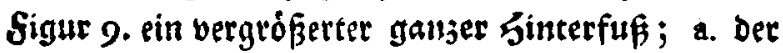

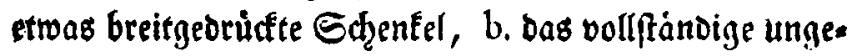

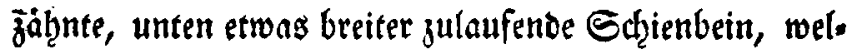

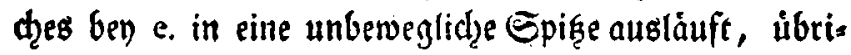
gens aber eine Rrone yon oren beweglidzen, blos einges feģten ḩarten ḩornartigen Spiz̧en ḩat. e Der eigentis he Fuß̧, wie vorhin. Die einzelnen F̧uß̧blätter fino an if̧ren Spif̧en ftarf, oben rund, unten aber breit, und b̧aben ein jedes, auf jeber Seite eine beweglidze Spize.

Siguv 10 , ein vergrókertes $G$ d) fenbein von einem weiblíden Melolontha Fullo, a. Ein Kheil beg Sdzen: Eels, e. bas Sdhienbein felbft, b. b. b. brev am $\mathfrak{A} u$ ffens

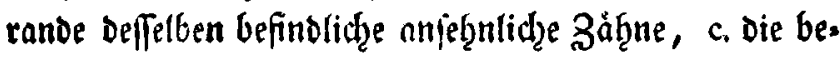
wegliche fteife eingefegte @piß̧e, d. bas erfte Glieb des Fufers.

Sig. I I, ein vergro̊fferter 弓́nterfuf Ses Weibdseng.

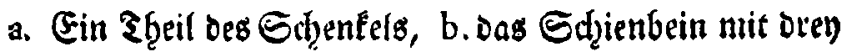
Dornen c. c. c. auf ber Uluffenfeite Deffeloen, wovon ber

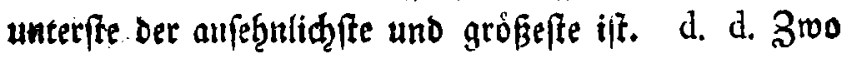
fteife bereglidie, auf Dem ßande der Srone eingeferte Gpifen. Bon Den frủien lę̧e man alle oben bev) Dex Battung Scarabaeus aus Fabr. Phil, Ent. angezogene §. S. nad̨. 
Anmertung. Th wirbe die lef̧ten benden Figuren ganj weggelaffen ḩaben, wenn die Füßje, ich möhte faft fagen aller weiblidzen 9Melolontlęen, nichet allju fệe bon jenen ber mánnlid̨en verfąieden wären, weldhes die gegeneinander ju Galtenden $\mathfrak{A b b i l d u n}$ gen berfelben an auffallenoften oarftellen merden. IIm nidzt eine Gadze zu oft zu wiederţolen, b̧abe

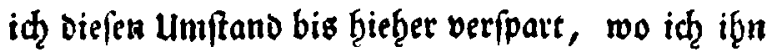
auf einmab̨l anjeigen fann. Bemóh̨nlidbermeife findet man fonft bey Den mànnlichen Ráfern frár. fere Bliedmấen und Şaffen, als bey den weibli, deen; aber bey biefer Battung /heint bie Natur entgegengefeģt verfaberen $\mathfrak{u} \mathfrak{u}$ ḩaben, wenigftens in Abfidze auf bie Füßze, reil die Edyienbeine bey)

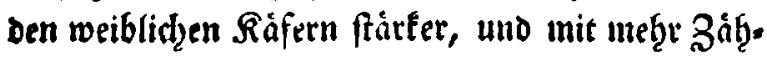
nen berwaffnet fino, als bei) den månnlidzen, uno bies nicht allein an den borderen, fondern aud an Den benden hinterfen Panren, wie foldzes unfere Figuren 8. 9. 10 und I . Deutlid, naḑweifen. Gollte man aber fragen: warum die Natur

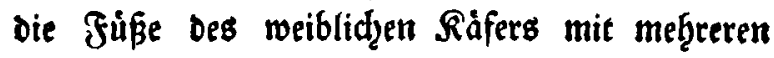

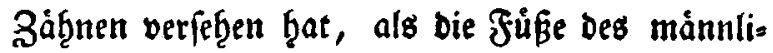
chen? fo wäre ich beynabe geneigt, ocsauf ju ants worten: weil Das $\mathfrak{B}$ eibdzen foldzer, ju Einbrin. gung in bie mor/dzen Baumftàmme und พurzeln, um bajelbft den abzulegenden Saamen mit Eidzer. beit unterjubringen, mef̧e beno̊ţ̧igt ift, als bas Männḑen, bem eine fo wid̨tige Naturpflidzt nidzt

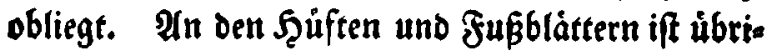

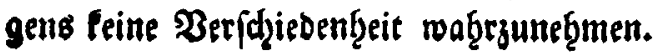


III. Dic 3erglieberung sinez Z゙äfers aus ber Gattung Scarabaeus Linn. (Cetonia Fabricii), nảmlid der Ce. tonia aurata. Fabr. Gen. Inf. p. 9. Gen. 6.

Os maxillis palpisque quatuor.

Sigur 12. ber aufebulid) vergróferte Ropf nebft Sem Bruftidilbe. a. a. Der Ropf felbft, b. ber vore Dere ftart ausgefdyweifte $\Re$ ano, c. c. Die braunen feb̧r

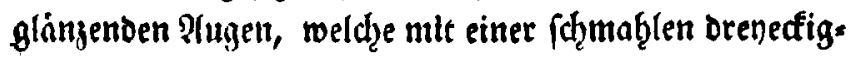
ten Rante umgeben find, d. d. bie Fưhthiçơrner *), welḑe aus zeţn ungleidyen Giliedern beftehen. Das erfte iff

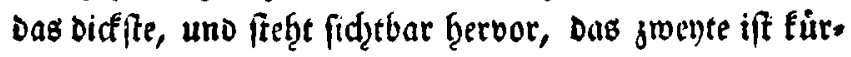
zer, uno meniger bict, die folgenden fünf faft forallen= fơrmig, unb von gleidzer Brößje, Dann die aus bren $\mathfrak{E} a$ s inellen befteţende Reule ober Rnopf, oeren mitteffie über Den ůbrigen benden ein wenig berbor ffeht, und alfo die länglte iff. Die Fưb̨lbeörner fino, bis auf ben Rnopf, gagat/ḩwarg, mit einem matten grünen @ḑimmer, Diefer aber fällt ber Farbe nad oft ein wenig ins graus. liḑe, Fabr. Gen. Inf. p. o. Gen. 6. Cetonia. Antennae breues, clanato lamellatac; lamellis tribus ouatis, articulo primo porrecto, craffiori. e. e. Die beyoen

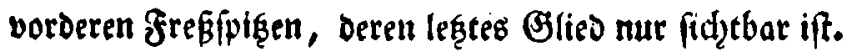
(Degeer IV. Taf. I I. Jig. 2.) f. f. Der verfdiedent=

$$
\text { c } 3
$$

lidę

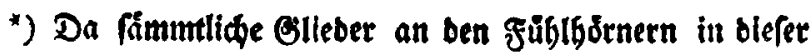
Bergrd derung beutlid gu erfennen finb, fo Galte id) eine anfebnlidhere fúr áberflübig, und bemerte nod, bas fie ben allen mir betannten నáfern btefer Battung bes Sabrieius. von gleider Geftalt finb. 
lid, artig aber fanft autsgefdzweifte Bruffldilto, welder eben fowob̨i nls ier Sopf, mit cinem feinen erb̧abenen Rande numgeben ift.

Sigur I 3. Sie febe vergro̊ferte Oberlippe *). Dies fe ift ăußerlich gar nid̨t zu bemerken, fondern fiz̧t un. mittelbar bid̨t unter bem Ropfidiloe, ift dưn, flach, ziemlich hart, uno bráumlich. Eie bat fomohl am Ran. be als auch all der ganjen fläbe, wo fich gleidefam auf jeder Seite eine Afrt bon Ueber/chlag zeigt, feḩt viele fudzbrothe Şaare, unter waldzen einige ungemein bif uno freif, uno alfo borftenartig fint. Fn ifrem Bruno. theil a. a, wofelbft fie an ben Ropffdhilo vernioge ftarker Mubfeln befeftige iff, faun fie von bem şier beroagt werben, vorn aber juifden b. b. ift fic fehre allsgefdenit=

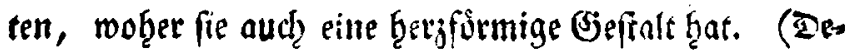
geer IV. ₹af. 11. Jig. 3 .

Sigur I4, Sie vergro̊ferte Interlippe Fabr. Gen. Inf. p. 9. Gen. 6. Cet. Labium elongatum, coriaceum, cylindricum apice fifum, aut emarginatum, palpos pofteriores fere totos tegens, mit allew ibien Theilcu, nainlidi a. a. bie Sippe felbft, weldze lederartig, worn faft gefpalten, noer viclmeger frarf ausegeranbet, mit langen gologelben Sgaren umgeben ift, und bie

*) Da ith biefen Ibeil fatjon ben bem Scarabaeus mit Des geer Duta) biefen $\mathfrak{R a b m e n}$ bezeichnet babe, fo will id) ibn alth bier belsefgalten. Sian fefe Tab. A. fig, I2. und Esite xxir. Şig. 12. in Der Ertlắrung. 


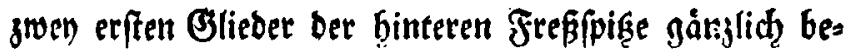
Dedft. b. b. Die inneren Rinnlaben (Maxillae Fabr. Gen. Inf. p. 9. Gen. 6. Cet. Maxilla ad infertionem palporum dilatata, vnidentata, fetofa), Deren jede aus zwen Sheilen beftef̧et, bem oberen 'c. c, Det einen feţe langen gologelben fearbuifhel vorftellt, bennod, aber ein braunes ḩornartiges Pláttchen ift, woran biefe lans gen f̧aare fikęen, unb Dem unteren b. b, weldher ungleidr viel gróker, sunfelbrautn ift, unb auf ber fichtbaren flá

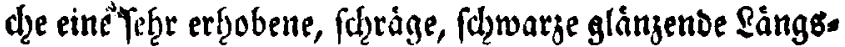
fante bat, ủbrigens ober aud auf allen Seiten fef̧r be: beare ift. SIn biefer firgen auch bie vorderen viergliebrigs

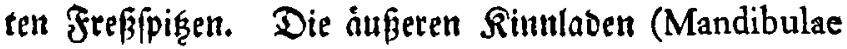
Fabr. Gen. Inf. pag. 9. Gen. 6. Cet. Mandibula porrelta, cornea, recta, acuta, inermis,) ben d. d. fint biel Eleiner als die inneren, hornartig, und ebenfalls mit J̧aaren runo ḩerum befȩ́t, (Degeer IV. Taf. I I. Fig. 4 - 7 .

Sigur 15. Die fdon erwåbnte åupere Kinmlabe Felgr vergrófert *). a. a. Die eigentlidze Riunlabe jelbft, wie fie fabricius bejd̨reibt, ţervorragent, ţorn:

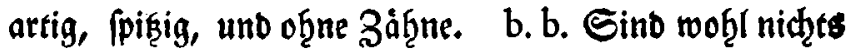

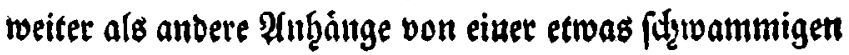

$$
\text { c } 4
$$

Rotve

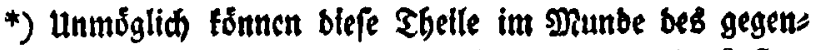

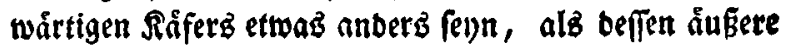

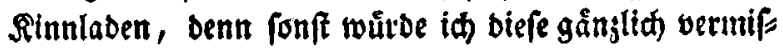
fen, wefl id in (ed)s zetbrodjenen నafern burdjaus tele ne andere finben fonnte, uno sabricius ifnen bod bee: gleiden Frefiperfseuge jueignet. 
Ronfiftent, bie eigentlidz̨ niḑ̧ zut Rinnlabe geḩdren. (Degeer IV. Taf. I I. Fig. G.) Fabr. Gen. Inf. I. c.

Sigur 16. cine felst vergrofferte vorbere frepfipitse. Sie beftę̧t nur aus Dren Bliedern, Deren leģteres a. Das langite, und walgenformig ift. Sie fino an Den inne: ren Rinnladen befeftigt. Fabr. Gen. Inf. p. 9. Gen. 6. Cct. Palpi fubaequales, anteriores filiformes, triar. ticulati: articulo vltimo cylindrico adhaerentes maxillae.

Sigur 17. eille ber biuteren Sreffpisen felye vers grSfert. Sie find ebenfalls orengliederidzt, bas oritte a. am lánglten, und ein wenig biffer als bie beyben er:

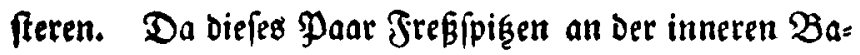
fis ber Unterlippe befeftigt ift, uno von berfelben beyna: Qe gänlid bedect wirb, fo ift bavon, wenn fie nods batan figen, nur das vorberfte BJtied fichtbar. Fabr. Gen. Inf. p. 9. Gen. 6. Cet. pofteriores (palpi) triarticulati: articulo vltimo longiori, fubcraffiori bafi internae labii inferti.

Sigur I 8. Die eigentlid) $3 \mathfrak{n}$ ft (Pectus) von unten in natúrlidjer Groffe. Diefer Ş̧ail bat bey oer gegen: wärtigen (Battung *) etwas eben fo merfwüroiges als abzeid:

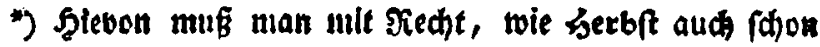
getban, verifjiebene Itrten von ber Gattung Cetonia bes Sabricius, bie, wenn fie nadh gennuer ju prifien. ben Renmeid)en, wo nicht ein eigeneś Genus aušmad)en, boch eber unter Mclolontha ju fetllen weiten, alş unter Ce- 


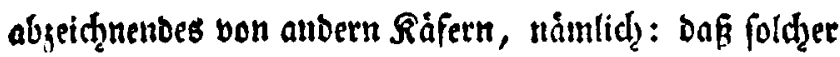
vorwärts in ein runtes bৃohess $\mathfrak{B r u f t b e i n}$ ausgef̧et, tefs fen Ende ein rundes Snỏpfchen a. zwifden ben $\mathfrak{B}$ urgeln Der Mittelfüpe, befindlid, ift ; binterwátts aber fid zu

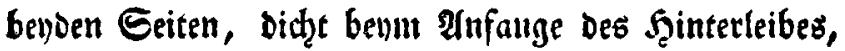
berlángert, und nuf jeber Seite eine am Sörper beerbor. tretende Epig̨e c. c. bilbet, woourd, wenn man bent Sáfer von oben betradztet, gleidzfam gren Tebenanb̨ăn.

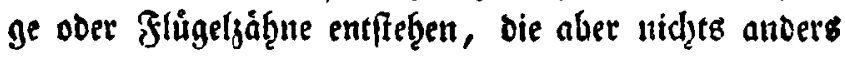
fino, als eben biefer herbortretende Siand ber unteren Bruft. Ueberdem befinden fich oben an ber $\mathfrak{B r u f t}$ zwo tleine gemölbte, Gefonders angefegete Platten a. a, weldhe insbefonbere auf Der Sberfeite fidtbar find, und ůber

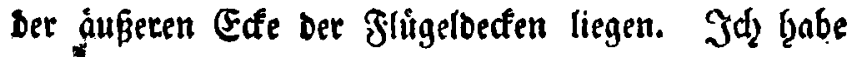
Figur 18. A. eine foldze P̣latte nadi iţern gaujen lım: fang uno Beftalt bergro̊ Bert vorgeftellt, win i̧̧re Sage genauer beftimmen zu fönnen. E\& if Daran a. Diejenige Rante, die man von dben Feḩen fann, b. ḩingegen Diejes nige, weldze von unten fidztbar ift, c. Dag aufizere Ende oder bie Spike, uno d. Die $\mathfrak{B} a[i s$, wo Die Platte mit ber f̧ornartigen $\mathfrak{B e r l a ̈ n g e r u n g ~ d e r ~} \mathfrak{B r u f t}$, welche in ben Bruftfdjilb einfugt, verbunden iff. Diefe fann, fo lange

$$
\text { c } 5 \text { Brult. }
$$

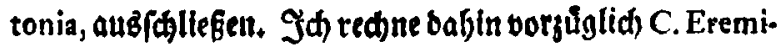
to, C. octopunctata, unb C. nobilis, unter Den befannton,

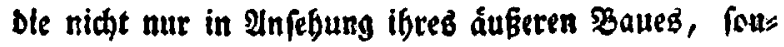
bern auch wegen Sangel bes gebacten Bruftueines,

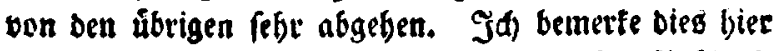
nur einftweilen, werbe aber in ber Folge, bei) Befdrei= bung Der einjelnen Mrten, meine NRelnung Daruber aue: fibilidger borttrigen. 
Bruftidilto uno $\mathfrak{B r u f t}$ mit einander verbunden fint, nidye geferten werben, fondern es bleith alsbann blos bet Sheil a. b. c. vou ber Platte fid thar. Diefe Platte ver=

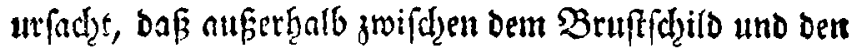
Jlügelbeclen feine Einlenfung, wie bey andern $\Re$ äfers Battungen, entftęet, fonbern baß es vielmeţr fdeint, als ob bende bicht aneinatider lägen, oder vielmehre, als ob die äuß̧eren Eifen Der Flúgeldecten fo weit berlängert wáren, daf fie fo bid̨t an Die Jlhigerbeden anpaßten.

Sigur 19. ein vergróferter Dorberfü, a. Fin

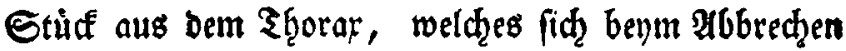
Des Sußzes mit abgelöpt, b. Der eigentlid̨e Schenfel,

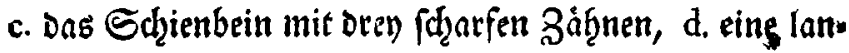
ge, ḩarte, eingejegete und berwegliche Spig̨e, wie bey Den vorigen, e. Der $J u \beta$, vorn mit jwo Rlauen verfę̧en. Die Şüfte ift faft burdzgångig mit langen feinen $\mathfrak{C}_{a}$. ren bewadchen. Sowohl Schentel als Sdchienbein fint

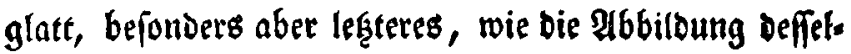
ben Deutlid, zeigt.

Sigur 20, einer von ben Mittel. Oder Żufffîfen

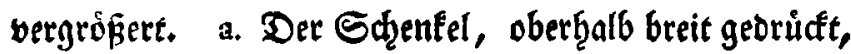
ums Sinie aber rundlidzer, b. Das Sdzienbein, auffer. நalb die Rante abgerundet, mit zwey anfef̧nlidenen Dorn. fpigen c. c, innerţalb bey f. flach unb groenfanticht. Unten an Der Rrone jwo berweglidze lange Spizen d, und nàdhftom nod, auf jeder Seite einen aus bem

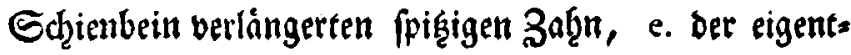
linge Juß. 


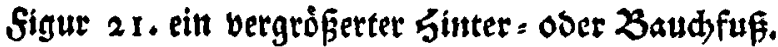
a. Eine Platte, ober ein Segment aus dem f̧interleib, welches fich benm Inareifien abgelófjt, b. oer eigentliche Sḩenfel, von befonderer Beftalt, uno meţe ovaltund

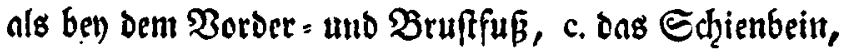
mit abgerundeter Pufenfante, woran wieberum jro ans

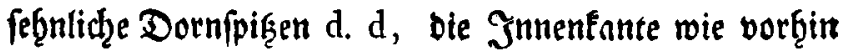
flach, unten an berfelben bel) f. ebenfalls zwo berweglidze lange Spizen an ber Rrone, uno zwen unbeweglidze aus bem Schienbein verlängerte fpişige Zảhne. g. Der Fuß̧, hier etwas gróker unb ftàrfer als an ben erfteten zwel) Paaren. Aluch hicrben faun man bie, bey Den F̧ůżen Des Scarabaeus aus Fabr. Phil. Ent. ongezogene

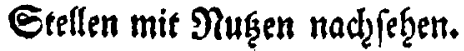

\section{$\mathfrak{N a \mathfrak { a }} \mathfrak{t} \mathfrak{r} \mathfrak{a} \mathfrak{g}$.}

Lim boch etwas bon ben ausgelaffenen benden (5)ats tungen Trox uno Trichius Fabr. beuzubringen, weil fie bende unter bes Linme Scarabaeus fteden, bqabe idh mir

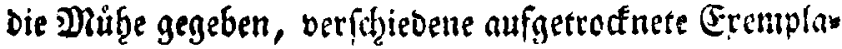
re zu erweidzen, unb - za zerbrechen, leioer! fann ids woḩl fagen, beng id zerbrad feḩr viele fruchtlos, unto

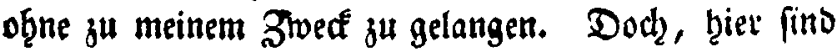
Die fragmente, womit fid die Riebtzaber fo lange be= gnůgen múffen, bis ish wiederum ourd frifde Erents. plare in ben Stand gefegt merde, meine Unterfudzhlt. gen ůber Die Jreprertgeuge Diefer benden Sattungen weiter fortzufegen. 